\title{
Visual Self-Misperception in Eating Disorders
}

\author{
Stephen Gadsby \\ Department of Philosophy, University of Antwerp, Antwerp, Belgium. \\ ORCID: 0000-0002-2302-844X \\ Forthcoming in Perception \\ Please cite published version
}

\begin{abstract}
Many who suffer from eating disorders claim that they see themselves as "fat". Despite decades of research into the phenomenon, behavioural evidence has failed to confirm that eating disorders involve visual misperception of own-body size. I illustrate the importance of this phenomenon for our understanding of perceptual processing, outline the challenges involved in experimentally confirming it, and provide solutions to those challenges.
\end{abstract}

\section{Introduction}

A common complaint from those who suffer from eating disorders - both anorexia nervosa and bulimia nervosa - is that they see themselves as "fat". This complaint has been a feature of clinical reports since the 70s (see: section 1), leading at least some contemporary researchers to assume that visual misperception of own-body size is a central feature of eating disorders. For example, Brooks and colleagues $(2016$, p. 1) write that people with eating disorders "often view themselves as much fatter than they really are". Similarly, one clinicians' handbook states, "In [anorexia nervosa], a sufferer does not see her skeletal body as it really is ... she does not just think she is fat, but also sees ... herself as fat" (Mondraty \& Sachdev, 2011, p. 3257, their emphasis).

Despite some scientists assuming that people with eating disorders visually perceive themselves as larger, this claim has yet to be empirically confirmed. ${ }^{i}$ While studies dating back to the 70 s provide compelling evidence that people with eating disorders misestimate their own body size (see: section 3), the question of whether they also visually misperceive their own body size has yet to be answered. In this paper, I outline some of the problems that researchers face in using behavioural tasks to test for the presence of visual selfmisperception in participants with eating disorders, and I describe how experiments can be designed to overcome such issues.

In section 1, I introduce the hypothesis that people with eating disorders visually misperceive their own body size, distinguishing it from some similar hypotheses. In section 2 , I outline some implications that the hypothesis holds for our understanding of perceptual processing. In section 3, I discuss prior attempts at experimentally confirming the hypothesis, involving tasks that require participants to visually estimate the size of their own bodies. I introduce three challenges that researchers face in using these tasks to confirm the presence of visual self-misperception. In section 4, I discuss some variations of body size estimate tasks and illustrate how they relate to the three challenges. Finally, in section 5, I discuss solutions to the challenges, in the way of experimental design principles.

\section{The Visual Self-Misperception Hypothesis}


The idea that eating disorders involve visual self-misperception can be traced back to the well-known clinician Hilde Bruch (1974, p. 89), who claimed that anorexia nervosa involves a "disturbed size awareness". Bruch based this claim on observations of her own clients and their complaints about perception of their own body size:

...even after a good therapeutic relationship has developed, when they appear to be actively interested in understanding the background of their condition, they will complain, with a certain bewilderment, that they cannot "see" how thin they are (ibid., p. $89-90)^{\mathrm{ii}}$

Beyond Bruch's work, complaints about misperception of one's own body size have featured in several first-person reports (Espeset et al., 2011; 2012). I will refer to the claim that people with eating disorders visually misperceive their own body size as the visual selfmisperception hypothesis. Understanding this hypothesis, and how it can be experimentally confirmed, is the aim of this paper. First, I will discuss a few features of the hypothesis.

The first thing to note is that visual misperception in eating disorders is assumed to be self-specific, only affecting perception of one's own body size. People with eating disorders do not report misperception of other people's bodies (Smeets, 1997, p. 78), indeed, they are often surprised at just how thin their peers appear (Espeset et al., 2011, p. 185). While some contemporary researchers have proposed that eating disorders involve domain general misperception of body size, I will not address this hypothesis here (Brooks et al., 2019).

It is important to distinguish the visual self-misperception hypothesis from some related hypotheses with which it is sometimes conflated. The first pertains to cognitive differences associated with eating disorders. Consider the experience of seeing a banana: when looking at them, bananas appear to us as a certain size, shape, and colour. We can distinguish this perceptual experience from the cognitive states - thoughts, judgments, and beliefs - that we form about bananas, such as the judgment: "that looks delicious", or the belief "there is a banana in front of me". The visual self-misperception hypothesis is not about the kinds of cognitive states (thoughts, judgments, and beliefs) that people with eating disorders form when they look at themselves, it is about the way in which they perceive their bodies.

Research shows that eating disorders are associated with negative judgments and beliefs about body size (Cash \& Deagle, 1997). Researchers are attempting to understand why such judgments occur. They might occur due to visual misperception - wherein those with eating disorders see their bodies as larger-or they might occur despite accurate perception of body size. For example, people with eating disorders might simply apply particularly harsh standards, judging themselves as "fat", "wide", or "overweight" where others would not. The important question, then, is what these individuals see when they look in the mirror, not what they think, judge, or believe.

The visual self-misperception hypothesis must also be distinguished from a hypothesis about attentional differences. Evidence suggests that, when looking in the mirror, people with eating disorders selectively attend to the (self-rated) "unattractive" parts of their own bodies (Tuschen-Caffier et al., 2015). While this difference in attention does change their perception of themselves - they perceive fatter as opposed to thinner parts, in virtue of attending to those parts-it is also not what is at stake. The visual self-misperception hypothesis claims that when people with eating disorders view themselves (for example, in a mirror), the body parts that they attend to are misperceived, appearing as larger.

First-person reports support this strong interpretation, as many with eating disorders describe seeing their bodies as larger, in ways that cannot be reduced to judgments or thoughts about the body. For example, when asked what they see when they look at 
themselves in the mirror, many draw a distinction between what they see and what they think, or believe:

With my eyes I actually saw myself as big, but my intellect told me that it couldn't be true (Espeset et al., 2011, p. 184)

In the mirror I see that I'm fat, but I do actually understand that it can't be true (Espeset et al., 2012, p. 523)

Consistent with the visual self-misperception hypothesis, these reports distinguish perception of the body from judgments about the body. ${ }^{\text {iii }}$ While such reports do suggest the presence of visual self-misperception in eating disorders, they are anecdotal. As such, they should be treated with caution. My goal in this paper is not to make strong empirical claims based off anecdotal reports, rather, it is to address the possibility of verifying such reports with experimental data.

\section{Theoretical Implications}

\subsection{The Cognitive Penetrability of Vision}

Research into visual processing has been greatly advanced through studying cases in which it malfunctions. For example, cerebral achromatopsia (loss of colour perception) and motion blindness (loss of motion perception) were both instrumental in providing insights regarding the visual processing of colour and motion (McCloskey \& Chaisilprungraung, 2017). The possibility that eating disorders involve visual misperception of body size would be equally instrumental in understanding how visual processing of body size functions.

Note that the visual self-misperception hypothesis does not suggest that people with eating disorders exhibit a domain general form of misperception. Unlike cerebral achromatopsia and motion blindness, eating disorders are not suggested to involve an inability to perceive the relevant properties (body size). Rather, they are assumed to involve a qualitative difference in the way in which those properties are perceived, namely, as wider. Further still, this difference does not occur in all cases of body size perception, only in perception of one's own body size. It thus qualifies as an incredibly unique form of misperception, which may uncover surprising insights regarding the functional characteristics of visual perception. Indeed, the visual self-misperception hypothesis has ramifications for perhaps the most controversial issue in vision research: the cognitive penetrability of vision (Firestone \& Scholl, 2016; Stokes, 2013).

Think back to the distinction between perception and cognition. It is uncontroversial to state that perception causally influences cognition; seeing a banana induces various cognitive states, for example, judgments about its taste ("how disgusting") and beliefs about its presence ("there's a banana in front of me"). What is controversial, however, is whether cognition can influence perception, for example, whether believing "there is a banana in front of me" could cause one to see a banana. This possibility is referred to as cognitive penetration, and it comes in many forms, each of which is the topic of their own debates. Two particularly controversial forms of cognitive penetration involve beliefs influencing perception (Stokes, 2014) and emotions influencing perception (Niedenthal \& Wood, 2019).

Some first-person reports suggest that these two factors may be involved with visual self-misperception in eating disorders. Specifically, they suggest that emotional states and the expectation of self-viewing can modulate whether misperception occurs: 
Yesterday I was really sad and today I'm in a better mood. Today when I look in the mirror, I see myself differently, more positive. Yesterday I only saw a big hippo. Actually, I've never thought about it before, that I see my feelings in the mirror. I think that's exactly how it is; when I have a bad day, I always see a big hippo. (Espeset et al., 2012, p. 524)

I remember one occasion, I was passing an open door and saw myself in the mirror, but actually, I didn't know that I saw myself. I just saw the image of a person in the mirror and thought; "Oh gosh, she is thin!" But then, when I understood that it was actually me, I didn't see me as thin anymore. But then I actually saw a glimpse of it. (Espeset et al., 2011, p. 183)

As before, we ought to exercise caution in how much evidential weight we give to these anecdotal reports. Nevertheless, they suggest that, in the case of eating disorders, vision might be modulated by non-perceptual states, such as emotions or the expectation of seeing oneself. This possibility warrants further investigation and discussion (Gadsby \& Hohwy, 2020).

Even if we reject the possibility that visual self-misperception in eating disorders is caused by cognitive or emotional states - it might, for example, be underpinned by entirely perceptual mechanisms (Brooks et al., 2019) — it nevertheless represents a compelling form of aberrant visual processing, given its domain specific nature (i.e., being restricted to the dimensions of one's own body). Such a phenomenon may provide insights into how human bodies are visually processed (Peelen \& Downing, 2007).

\subsection{Tactile and Proprioceptive Body Representation}

In processing proprioceptive and tactile signals, our brains rely on perceptual representations of our own body size. In order to compute the distance between two points of tactile contact, the brain maps those points on to a representation of the skin's surface (Longo et al., 2010). In order to compute motor commands and control movement, the brain relies on a representation of the relevant body parts (Gadsby \& Williams, 2018). Mounting behavioural evidence suggests that, in the case of eating disorders, these body representations are distorted, representing the body as larger. In the case of touch, participants with eating disorders systematically judge tactile distances as larger than neurotypical controls (Keizer et al., 2011; Keizer et al., 2012), especially along salient body parts (e.g. the waist and abdomen) (Risso et al., 2020; Spitoni et al., 2015). ${ }^{\text {iv }}$ Similarly, when passing through apertures (such as doorways), participants with eating disorders move their bodies as if they were larger (Beckmann et al., 2020; Engel \& Keizer, 2017; Keizer et al., 2013; Metral et al., 2014). This evidence is taken to suggest that people with eating disorders exhibit tactile and proprioceptive representations of body size that are distorted (Gadsby, 2017a).

There is considerable debate over how perceptual body representations function (Gadsby, 2019). As with the study of vision, this research is informed by disorders in which body representations malfunction (de Vignemont, 2010). To understand how representation of the body functions, we must understand how and why it malfunctions in cases of psychopathology, such as eating disorders. The visual self-misperception hypothesis provides an answer: if people with eating disorders visually misperceive themselves, then this misperception may provide the inaccurate content exhibited by their body representations. Given that representations of body size are highly dependent on visual information (De Vignemont, 2018), if such information was inaccurate, then this would explain why these body representations become distorted. Consequently, the visual self-misperception 
hypothesis holds important implications for our understanding of tactile and proprioceptive processing.

\section{Visual Self-Misperception and Body Size Estimation}

Directly inspired by Bruch's clinical observations, researchers began attempting to verify the visual self-misperception hypothesis by conducting experiments requiring participants to estimate their own body size (Slade, 1985). The first of these experiments, by Slade and Russell (1973), instructed participants to manipulate the distance between two lights (mounted onto a track) until it corresponded to the width of different body parts (chest, face, waist, and hips). While the control group estimated body size accurately, the eating disorder group overestimated the width (but not height) of several body parts. These participants did not, however, overestimate the size of inanimate objects (see also: Bowden et al., 1989; Brown et al., 2021; Garner et al., 1976), and only slightly overestimated other women's body parts.

Over the past four decades, researchers have developed and employed many variations of these body size estimate (BSE) tasks. The results of these experiments suggest that, on average, participants with eating disorders (both anorexia nervosa and bulimia nervosa) overestimate their bodies compared to neurotypical controls (for review and theoretical discussion, see: Farrell et al., 2005; Gardner \& Brown, 2014; Mölbert et al., 2017; Smeets, 1997; Smeets et al., 1997).

Early on, researchers interpreted these results as confirmation of the visual selfmisperception hypothesis, assuming that participants estimated themselves as larger because they saw themselves as larger (Garner \& Garfinkel, 1982; Slade \& Russell, 1973). This assumption requires some unpacking. Because early BSE tasks restricted participants from viewing their own bodies, it is not immediately clear how visual self-perception could be implicated (Smeets, 1999, p. 46). Such tasks were not assumed to directly measure participants' perception of themselves. Rather, they were assumed to do so indirectly, via the medium of visual memory. Researchers assumed that in order to estimate one's own body size, participants would rely on a memory of their own body, often referred to as "the body image" (Schilder, 1935). Consequently, BSE tasks were taken to be a measure of the body image's dimensions - "if overestimation takes place, the body image has to be fatter" (Smeets, 1997, p. 88).

However, even if overestimation stems from a distorted body image, this does not entail distorted visual perception of the body. For that conclusion, an additional assumption is required, namely, that the content of the body image is exclusively derived from visual perception. Smeets and Panhuysen (1995) describe this assumption like so:

The body image was believed to be a snapshot of the body percept (the body as it is directly perceived), in which size and shape characteristics have been accurately preserved. Thus, overestimation of body size was interpreted as indicating how a patient perceives herself when looking in the mirror, the body image functioning as an intermediary station between this percept and the size estimate (p. 113, endnote 1)

In this way, BSE tasks were assumed be a valid and reliable measure of visual selfperception.

Throughout the $80 \mathrm{~s}$ and $90 \mathrm{~s}$, several researchers began to question whether these tasks could in fact provide evidence for the visual self-misperception hypothesis (for review, see: Smeets \& Panhuysen, 1995; Smeets, 1999). Here, I will discuss two concerns that arose. The first concern regards the role of additional factors in influencing body size estimates. The 
second concern regards the role of non-perceptual factors in influencing visual memory of the body. As I will demonstrate, these concerns are still relevant.

Consider the first concern. While participants may rely on their visual memory to estimate body size, other factors could also influence such estimates. Thinking back to the distinction between perception and cognition, body size estimates may be influenced by various cognitive factors. For example, participants may provide estimates of the body that they believe, think, or feel themselves to have, rather than the body that they perceive (Smeets et al., 2009, p. 158). Demand characteristics might also play a role, wherein participants overestimate in an attempt to "help" or "sabotage" the experiment (ibid.). The issue, more generally put, is that that there exists a strong possibility that responses in BSE tasks are biased by factors that researchers do not intend to measure. I will refer to this as the response bias challenge.

Consider the second concern. Even if body size estimates are directly reflective of visual memory - and those with eating disorders do remember themselves as larger than reality-we need not also assume that memory accurately reflects perception. Doing so would assume (as the above quote reflects) a notion of memory as operating much like a camera, storing faithful "snapshots" of experience for later recall. Researchers no longer hold such a view: memories are not so much recalled as reconstructed-a process which is itself prone to bias and error (Kourken \& Sutton, 2017). Many eating disorder researchers came to believe that this process of reconstruction was a more likely culprit for body image distortion (Smeets et al., 2009). According to this view, while overestimation may stem from distorted memory, the distortion occurs during reconstruction. I will refer to this as the reconstruction challenge. ${ }^{\mathrm{v}}$

Note that there is a straightforward way to avoid the reconstruction challenge: by measuring direct perception (rather than memory) of the body. There are different ways to achieve this, for example, by requiring participants to view themselves in a mirror (Shafran \& Fairburn, 2002), or by allowing participants to use a photograph of themselves as a reference point (Smeets et al., 1999). If participants follow instructions appropriately, such tasks will constitute a measure of direct visual perception and thus avoid the reconstruction problem. Consequently, much of this essay will be focused on how to avoid the response bias challenge, as well a new challenge, which I introduce in the next section.

\subsection{The El Greco Challenge}

El Greco was a Spanish renaissance artist who painted subjects with elongated forms. Some art historians suggested that this painting style was due to El Greco suffering from astigmatism, an ocular defect which causes a stretched-out perception of the world (Firestone, 2013). As Firestone \& Scholl illustrate, such an interpretation is fallacious:

If El Greco truly experienced a stretched-out world, then he would also have experienced a stretched-out canvas. In that case, the distortions should have canceled each other out: Just as El Greco would have seen real-word figures as elongated, so too would he have seen his paintings as elongated, and so the real-world distortions he experienced would never have transferred to his reproductions. (2014, p. 39)

This problem can be reduced to a simple principle, "When a constant-error distortion should affect equally the means of reproduction and the item reproduced, the effects should cancel each other out" (ibid., p. 45). This can be characterised in terms of the difference between one's perception of a stimulus and one's perception of the means of reproducing that stimulus, which I will label the input and output. If El Greco suffered from astigmatism, then 
both his perception of the world (the input) and his perception of the lines on his canvas (output) would be susceptible to the same distortion. Because of this match in distortion, the strokes of his paintbrush would remain faithful to the lines of the reproduced objects, and no distortion would be reproduced. If El Greco's perception was distorted, his painting would not reflect it. This presents a challenge for researching visual self-misperception: contexts where the relevant misperception is predicted to affect both input and output can undo evidence of misperception. I will refer to this as the el Greco challenge.

Note something about the el Greco challenge: it is not important what kinds of actions are used to reproduce the input. El Greco could be painting, drawing, manipulating a photograph, or answering a set of questions to reproduce a stimulus. So long as the same distortion affects both input and output, cancellation should occur. Consequently, the relevance of the El Greco challenge to BSE tasks is not determined by the kinds of actions participants take to indicate their body size. What does matter, however, is the appearance of the output. El Greco's assumed deficit (astigmatism) is domain general, causing him to perceive everything as elongated. Because of this, he would misperceive both the world and the canvas, leading to a cancellation effect. This is importantly different to the visual self-misperception hypothesis, wherein the relevant distortion only affects visual perception of one's own body size (along certain dimensions). This places an important constraint on how the El Greco challenge applies to BSE results. For cancellation to occur, the same distortion must apply equally to the input (the visually perceived body) and the output (the stimulus used to estimate the body). As I will illustrate, there is reason to expect cancellation to occur in many forms of BSE task.

\section{Body Size Estimate Task Variations}

\subsection{Stimuli Realism}

An important feature of BSE tasks is the kinds of stimuli used to estimate. For our purposes, the most relevant feature is their realism. In many BSE tasks, participants indicate their body size using stimuli that do not realistically depict themselves, such as silhouettes of bodies, computer generated models, or abstract distances indicated with lines, callipers, or points on a wall (for example, see figure 1). However, some BSE tasks employ photographs or videos of participants' own bodies, digitally manipulated to be different sizes (for example, see figure 2). Participants estimate their body size by manipulating the stimulus themselves (Collins, 1986), or by indicating a stimulus that most closely matches their own size. ${ }^{\mathrm{vi}}$

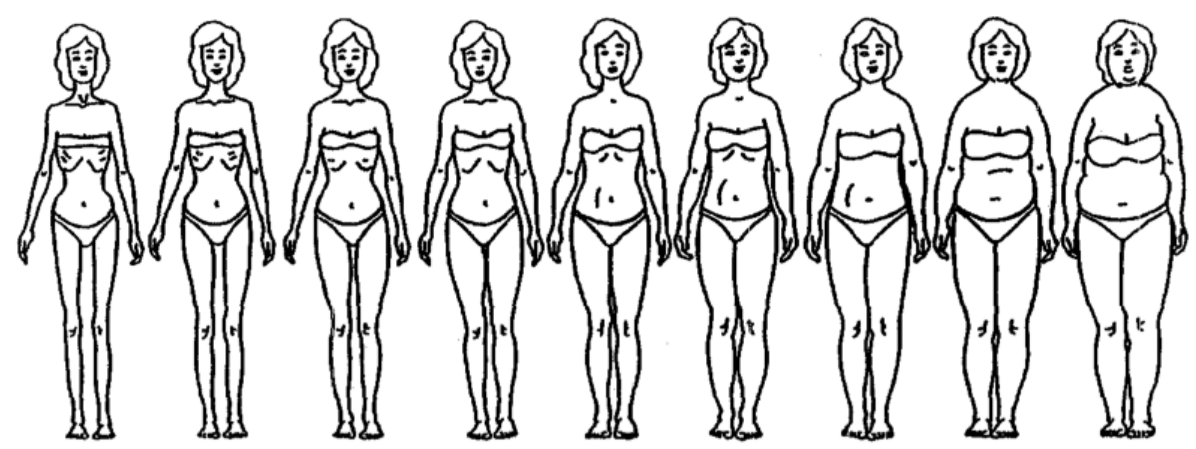

Figure 1. The contour drawing rating scale (Thompson \& Gray, 1995). Experiments that employ this scale instruct participants to select the image that most accurately depicts their current body size. 


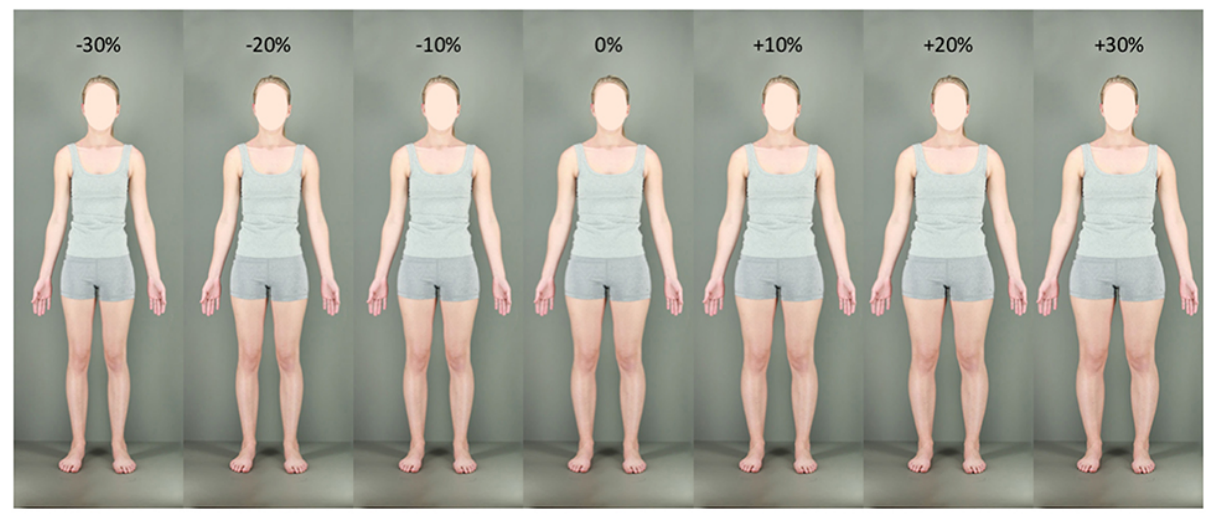

Figure 2. Realistic stimuli from a BSE task, created by manipulating a photograph of the participants' body (Brooks et al., 2016). Though removed here (for anonymity), the participant's own face is visible in the experiment.

The distinction between realistic and non-realistic stimuli is crucially relevant to the el Greco challenge. In the case of BSE tasks, the input is always perception of one's own body, whether directly or via the medium of visual memory. When the estimating stimulus is sufficiently realistic to induce misperception, the el Greco challenge predicts a cancellation effect. This poses a problem for the use of realistic BSE tasks to test the visual selfmisperception hypothesis. Consider two possible findings of a task that uses realistic stimuli.

Outcome 1: The eating disorder group overestimate their body size compared to the control group.

This outcome fails to confirm the visual self-misperception hypothesis. If participants truly misperceived themselves, then such misperception should cancel out, as both input and output would exhibit this distortion. Any misestimation, then, must stem from other factors (for example, response bias). Such an outcome also fails to disconfirm the visual selfmisperception hypothesis, as participants may misperceive themselves and the stimulus (leading to cancellation) but also overestimate their bodies due to other factors.

Outcome 2: No between-group differences in size estimation

This outcome also neither confirms nor disconfirms the visual self-misperception hypothesis. No between-group differences may be due to a cancellation effect occurring in the eating disorder group (consistent with the hypothesis) or because both groups accurately perceive themselves (contrary to the hypothesis).

The lesson for designing BSE tasks to measure visual self-misperception is that they must involve a mismatch between input and output. Otherwise, a cancellation effect will occur, and the results cannot be used to uncover misperception.

Note an issue regarding the el Greco challenge and stimuli realism. Given the lack of precisely specified theory regarding the cause of self-misperception in eating disorders, it is unclear when misperception should occur. For example, while people with eating disorders report seeing themselves as larger while viewing themselves in the mirror, it is an open question whether misperception extends to other visual representations, such as photographs. Without understanding more about how self-misperception in eating disorders functions, we cannot predict the kinds of stimuli that will induce it. Nevertheless, the possibility that stimuli are sufficiently realistic to induce misperception undermines the reliability of tasks that use 
such stimuli. The el Greco challenge can be avoided by ensuring that BSE tasks employ stimuli that are maximally non-realistic, for example, metric stimuli such as rectangles.

\subsection{Forced Choice Tasks}

Another important feature of BSE tasks is the procedure itself. In many BSE tasks, participants estimate their body size in a single trial. The estimate is compared against the participant's true body size, to calculate a measure of misestimation. In contrast, some BSE tasks show participants different sized stimuli over numerous trials and force them to make a choice between one of two responses: in most cases, whether the image is larger or smaller than their own body. While the last section argued that BSE tasks that employ realistic bodily stimuli are susceptible to the el Greco challenge, this section argues that - contrary to the claims of many researchers - these forced choice tasks are susceptible to the response bias challenge.

Forced choice BSE tasks were pioneered by Rick Gardner and colleagues in the $80 \mathrm{~s}$ and 90s (Gardner \& Bokenkamp, 1996; Gardner \& Moncrieff, 1988). These researchers sought to use principles from psychophysics to design tasks that could avoid the response bias challenge and determine whether overestimation of body size in eating disorders is due to misperception. As Gardner \& Moncrief (p. 106) put it, "Only by using a psychophysical methodology... can one investigate the separate roles of sensory and nonsensory factors". Forced choice tasks based on these principles are still used today, and contemporary researchers maintain the ability of these tasks to resolve issues related to response bias (Cornelissen et al., 2013).

Claims of distinguishing between sensory and non-sensory contributions to body size estimation are based on a distinction between two variables provided by such tasks: the point of subjective equality (PSE) and the just noticeable difference (JND). The PSE is derived by calculating the mean size of the stimuli in trials where the participant provides each response equally. As with measures of overestimation in standard BSE tasks, this variable is taken to be the "body size that an observer judges to be subjectively equal to their actual size" (Gardner, 2012, p. 526). In contrast, the JND is calculated as the difference between trials where responses are $25 \% \& 75 \%$, respectively, in other words, "the amount of change in body size necessary in order for an observer to detect that change one-half of the time" (ibid.) (see figure 3). The JND is interpreted as a measure of perceptual sensitivity: the ability to (visually) discriminate between two stimuli. 


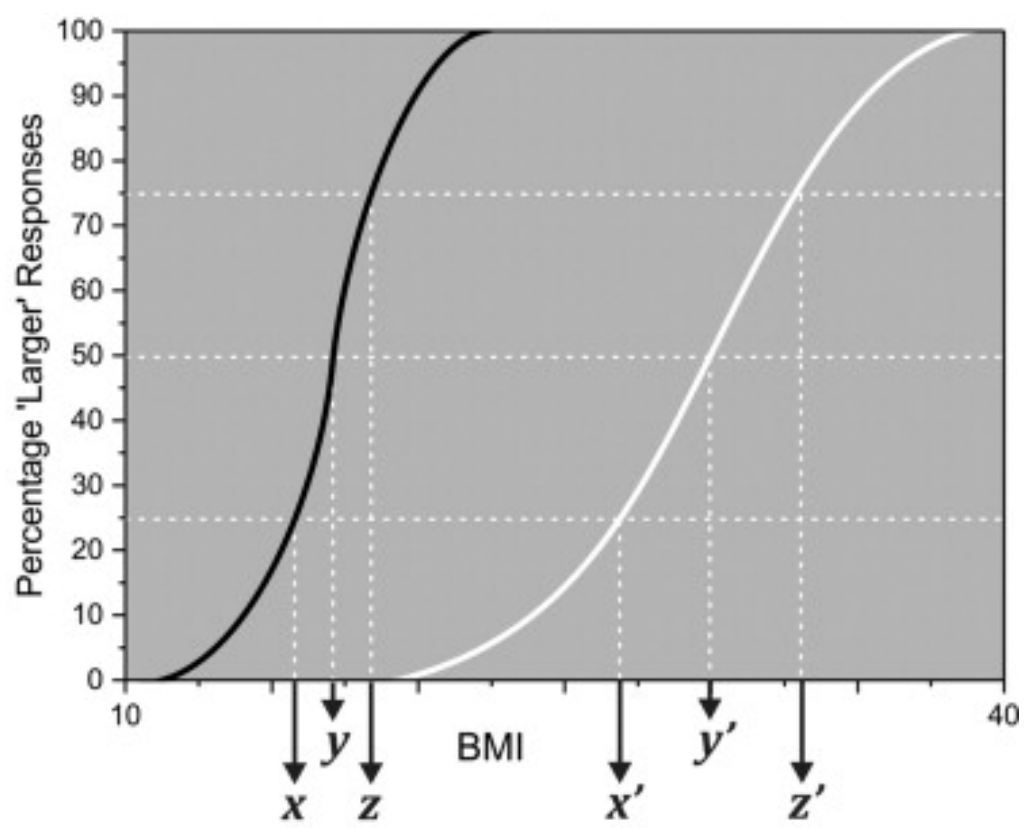

Figure 3. A plot illustrating the relationship between participant responses, PSE, and JND (Cornelissen et al., 2015, p. 82; reprinted with permission from Elsevier). Two hypothetical PSE values are represented, y and y': y represents a low body size estimate, y' represents a high body size estimate. The difference between $\mathrm{x} \& \mathrm{z}$ and $\mathrm{x}$ ' \& $\mathrm{z}$ ' correspond to two JND values, respectively. The former $(x-z)$ represents high perceptual sensitivity, while the latter $\left(x^{\prime}-z^{\prime}\right)$ represents low perceptual sensitivity.

According to researchers who employ these methods, the PSE reflects a form of response bias - i.e. "nonsensory, or affective, components of body image judgments" (Gardner \& Boice, 2004, p. 94), while the JND is a measure of the "participant's sensitivity in detecting changes in body size and is a relatively unbiased indicator of sensory factors" (ibid., p. 9394). Results of these forced choice studies indicate that participants with eating disorders exhibit differences in PSE (overestimation) but no differences in JND (for review, see: Garner, 2012).

Given their interpretation of the PSE and JND, many researchers consider these findings to have resolved the response bias challenge entirely. As Gardner (2012, p. 529) writes, "[results from these studies] conclusively demonstrated that it is cognitive factors and not any sensory factor that is responsible for anorexic individuals reporting they 'see' a fat image when they look in a mirror". This opinion is echoed in numerous papers employing these techniques, for example, in discussing the results of their forced choice task, Cornelissen and colleagues (2013, p. 110) write "[body size over-estimation] is primarily based on attitudinal factors (as indexed by PSE) rather than perception (as indexed by DL)."

It is straight forward to see that, independently, such tasks cannot be used to confirm or disconfirm the visual self-misperception hypothesis. First, note that, for this hypothesis, it is not relevant whether participants with eating disorders exhibit differences in perceptual sensitivity to changes in body size. The hypothesis pertains to differences in the dimensions that people with eating disorders perceive, when self-viewing, not their perceptual sensitivity. There is no obvious reason why misperception of bodily dimensions should necessarily be associated with a difference in body size sensitivity.

The second thing to note is that the PSE is not exclusively a measure of response bias. While it is likely that the PSE is susceptible to different forms of response bias, it is also determined by perceptual factors: most obviously, by the way in which the original stimulus 
is perceived. Consider a scenario where two participants, alike in all other ways, are shown the same stimulus, but one suffers from a visual defect causing them to perceive it as larger. When estimating the size of the stimulus in a forced choice task, the participant with the distorted perception would exhibit a larger PSE, as the perceptual reference point (the stimulus) is perceived as larger. Consequently, between-group differences in PSE can neither confirm nor disconfirm the visual self-misperception hypothesis, as any differences might be genuinely perceptual, or stem from response bias.

There are many useful features of forced choice BSE tasks, and these tasks have been put to good use in advancing several interesting hypotheses regarding the cause of body size overestimation in eating disorders (Cornelissen et al., 2013; 2015). While such tasks cannot currently answer the response bias challenge, future variations might. The literature on psychophysics features several methodological proposals for addressing similar problems, which may provide inspiration for future tasks (Jogan \& Stocker, 2014; Morgan, 2014; Morgan et al., 2013).

\section{Overcoming The Challenges}

In the previous section, I outlined several challenges for the use of BSE tasks to test the visual self-misperception hypothesis. In this section, I describe how these tasks can be designed to overcome these challenges.

Designs that rely on participants' memory of their own bodies are subject to the reconstruction challenge: different factors influence the reconstruction of memories, therefore misremembering one's body size is not evidence of misperceiving it. As noted, researchers can avoid this challenge by requiring participants to directly estimate their own body size (as many do).

For BSE tasks where both the input and output (the stimulus being estimated and the stimulus being used to estimate) are realistic depictions of the body, the el Greco challenge predicts a cancellation effect, and the results cannot be used to inform the visual selfmisperception hypothesis. This challenge can be avoided by creating a mismatch between input and output, such that the stimuli used to estimate are (entirely) non-realistic, for example, abstract lines or shapes.

While many variations of BSE tasks already avoid the reconstruction and el Greco challenges, the response bias challenge affects any task where participants are explicitly instructed to estimate their own body size. There are two main ways in which response bias undermines BSE results. The first is that participants' beliefs, thoughts, and emotions about their body size influence their response, for example, when they estimate the body size that they believe themselves to have. The second is that participants' assumptions about what the experimenter expects influence their response, for example, when they attempt to help the experimenter by providing the expected response. To avoid the first possibility, tasks must be designed to disrupt the predicted effect between body attitudes and body estimation. To avoid the second possibility, tasks must be designed to obscure the experimenter's expected response.

In what follows, I discuss one strategy for disrupting the link between attitudes and responses and obscure the expected response of the experimenter (for similar suggestions, see: Gross et al., 2014; Valenti \& Firestone, 2019). To provide an illustration of this strategy, I introduce the transposed BSE task. This task involves switching the input and output of a task in an unexpected way, requiring participants to estimate the size of a metric object by manipulating a realistic depiction of themselves (for example, a realistically sized photograph) (figure 4). 


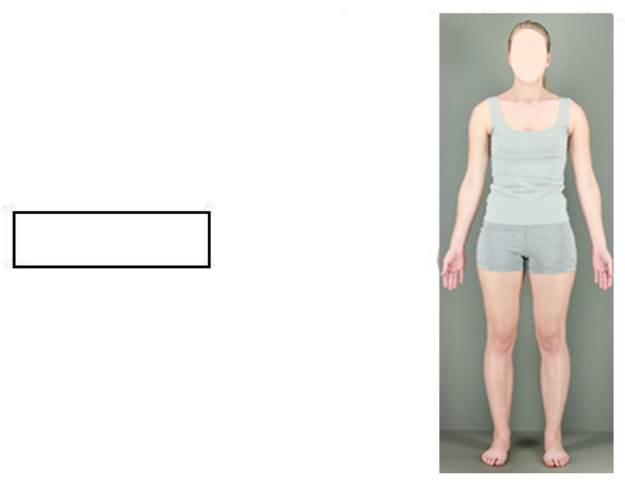

Figure 4. Example stimuli: perception of the rectangle is the input and perception of the photograph is the output. In the experiment, the participants own face would be shown. Potential task instructions: "manipulate the size of your body until the width of your abdomen matches the width of the rectangle". Photograph taken from Brooks et al. (2016).

First, note this task would avoid both the reconstruction and el Greco challenges. The experiment is designed to measure how participants (directly) perceive the stimulus used to estimate the rectangle (the photograph of themselves), therefore visual memory is not involved. Furthermore, there is a mismatch between input (rectangle) and output (photograph), so no cancellation effect is predicted. ${ }^{\text {vii }}$

What is notable about a design like this, however, is that it lessens the potential for response bias by both obscuring the experimenter's expected response and disrupting the predicted link between bodily attitudes and size estimation. It does so by predicting an unintuitive result: that participants with eating disorders will underestimate the size of the rectangle. To see how this works, consider two possible outcomes of this task:

\section{Outcome 1: No between-group differences in size estimation}

This outcome suggests that, just like the control group, people with eating disorders accurately perceive both stimuli and accurately estimate the size of the rectangle, without response bias. This finding is consistent with the hypothesis that there are no perceptual differences between these groups, disconfirming the visual self-misperception hypothesis.

Outcome 2: The eating disorder group underestimate the rectangle's size, compared to the control group.

This outcome provides evidence in favour of the visual self-misperception hypothesis. To see why, imagine that both stimuli (rectangle and body) are $20 \mathrm{~cm}$ wide. If participants accurately perceived the rectangle but misperceived their own body-say, as $25 \mathrm{~cm}$ wide, rather than 20 - then, in attempting to match the perceived size of the body to the perceived size of the box, the participant would need to manipulate the body to be (objectively) smaller.

Note how this outcome helps to dampen the response bias challenge. Given that the task does not require participants to estimate their own body size, participants will not overestimate due to selecting a size corresponding to how they believe, think, or feel about their bodies. ${ }^{\text {viii }}$ Similarly, it is unlikely that demand characteristics would cause such an outcome: it would require extremely careful reasoning on behalf of the participant to conclude that the experimenter desired them to underestimate the size of the rectangle. Because this desired outcome is much less obvious, the experiment is less susceptible to demand characteristics. ${ }^{\text {ix }}$ 
The transposed BSE task is presented here as proof of a general principle: by disrupting the link between bodily attitudes and body size estimation, and obscuring the expected response of the experimenter, BSE tasks can be made less susceptible to the response bias challenge. Consequently, they may still prove useful for testing the visual selfmisperception hypothesis.

\section{Conclusion}

I discussed the hypothesis that people with eating disorders visually misperceive their own body size. While first-person reports suggest that visual self-misperception occurs, BSE results have has yet to confirm or disconfirm these reports. My aim is not to undermine the general validity or usefulness of these tasks. ${ }^{\mathrm{x}}$ Rather, my aim is to assess the possibility that they can generate data to either confirm or disconfirm the visual self-misperception hypothesis.

I identified three methodological challenges which stand in the way of testing the visual self-misperception hypothesis. Two of these challenges - reconstruction and el Greco - can be overcome by requiring participants to directly estimate their body size and ensuring that there is a mismatch between input (the stimulus being estimated) and output (the stimulus used to make the estimate). The issue of response bias is more difficult to overcome and applies to any form of BSE task where participants are explicitly instructed to judge their own body size. To address this issue, I suggested that BSE tasks be designed to obscure the experimenter's expected response and disrupt the link between bodily attitudes and size estimation. I discussed one way of achieving this: the transposed BSE task, wherein the input and output are switched, and participants are required to estimate the size of an object by manipulating a photograph of themselves. By using a method such as this, there is hope for confirming whether body size overestimation in eating disorders is due to misperception or response bias.

The outcome of this research program has crucial ramifications for our understanding of perceptual processing. If visual self-misperception occurs in eating disorders, then the phenomenon can be used to inform models of tactile and proprioceptive body representation and may also constitute evidence in favour of the cognitive penetrability of vision.

\section{Acknowledgements}

Thanks to members of the Cognition and Philosophy Lab and the Dijkerman Lab for helpful discussion. Thanks to Manja Engel, Jakob Hohwy, Dan Williams, Jennifer Windt, and four anonymous reviewers for written feedback. This research was supported by an Australian Government Research Training Program (RTP) Scholarship.

\section{Endnotes}

${ }^{\mathrm{i}}$ While once widely held, the assumption that people with eating disorders visually misperceive themselves fell out of favour during the 90s (for a historical overview, see: Smeets, 1997). It is difficult to estimate how common the assumption is amongst contemporary eating disorder researchers. However, there are undoubtedly several research groups who hold it, for example, those attempting to illustrate that visual misperception in eating disorders is a result of perceptual adaptation (for a review, see: Brooks et al., 2019). In this paper, I argue that it is an important hypothesis not because it is widely held, but because it is strongly suggested by first-person reports (section 1) and bears important theoretical implications (section 2). 
ii This excerpt is followed by an anecdote:

A woman of 20, who seemed to be making good progress, admitted "I really cannot see how thin I am. I look into the mirror and I cannot see it; I know I am thin because when I feel myself I notice that there is nothing but bones"

iii One might argue that these reports do not distinguish differences in visual perception from differences in attention. However, it seems unlikely that one would report seeing themselves as larger when in fact they were simply attending to those parts of their bodies that they judged to be larger.

iv For some recent contradictory findings, see (Engel et al., 2021)

$\checkmark$ This challenge can be formulated more generally: for any BSE task that requires participants to estimate their body size without seeing themselves, participants must rely on an alternative representation of body size. The reconstruction challenge, more generally put, involves the possibility that this representation is distorted by factors distinct from visual perception.

vi The distinction between non-realistic and realistic stimuli is importantly different from the more commonly discussed distinction between metric and depictive stimuli (Mölbert et al., 2017). Metric stimuli are metric standards (e.g. lines or calliper distances), while depictive stimuli depict a human body (Longo \& Haggard, 2012). While metric stimuli all qualify as non-realistic, some depictive stimuli do also. For example, silhouettes or drawings of bodies are both depictive and non-realistic. The category of realistic stimuli I introduce here refers to a subset of depictive stimuli: those that depict realistically (such as photographs and videos).

vii To induce misperception, the photograph of the participant's body must be maximally realistic, for example, by appearing like a mirror reflection (Shafran \& Fairburn, 2002)

viii While the suggested task design does avoid the most discussed form of response bias (participants selecting body sizes that reflect their beliefs, thoughts, and emotions), it does not entirely avoid response bias. For example, unpleasantness associated with seeing their own bodies enlarged may bias participants towards manipulating the photograph to be narrower. Thanks to an anonymous referee for this suggestion.

ix This leaves a third potential outcome: the eating disorder group overestimate the rectangle's size, compared to the control group. While it is not clear why this outcome would occur, it is consistent with influence from demand characteristics, wherein participants incorrectly guess the experimenter's expectation (that appearance of one's own body causes one to overestimate).

${ }^{x}$ In fact, I have argued elsewhere that the process of self-comparison that occurs in these tasks may itself be a theoretically and clinically important phenomenon (Gadsby, 2017b). 


\section{References}

Beckmann, N., Baumann, P., Herpertz, S., Trojan, J., \& Diers, M. (2020). How the unconscious mind controls body movements: Body schema distortion in anorexia nervosa. International Journal of Eating Disorders.

Bowden, P., Touyz, S., Rodriguez, P., Hensley, R., \& Beumont, P. (1989). Distorting patient or distorting instrument?: Body Shape disturbance in patients with anorexia nervosa and bulimia. The British Journal of Psychiatry, 155(2), 196-201.

Brooks, K. R., Mond, J., Mitchison, D., Stevenson, R. J., Challinor, K. L., \& Stephen, I. D. (2019). Looking at the figures: visual adaptation as a mechanism for body-size andshape misperception. Perspectives on Psychological Science, 15(1), 133-149.

Brooks, K. R., Mond, J. M., Stevenson, R. J., \& Stephen, I. D. (2016). Body image distortion and exposure to extreme body types: contingent adaptation and cross adaptation for self and other. Frontiers in neuroscience, 10, 334.

Brown, T. A., Shott, M. E., \& Frank, G. K. (2021). Body size overestimation in anorexia nervosa: Contributions of cognitive, affective, tactile and visual information. Psychiatry Research, 297, 113705.

Bruch, H. (1974). Eating disorders. Obesity, anorexia nervosa, and the person within. Routledge \& Kegan Paul.

Cash, T., \& Deagle, E. (1997). The nature and extent of body-image disturbances in anorexia nervosa and bulimia nervosa: A meta-analysis. International Journal of Eating Disorders, 22(2), 107-126.

Cornelissen, K. K., Bester, A., Cairns, P., Tovée, M. J., \& Cornelissen, P. L. (2015). The influence of personal $\mathrm{BMI}$ on body size estimations and sensitivity to body size change in anorexia spectrum disorders. Body image, 13, 75-85.

Cornelissen, P. L., Johns, A., \& Tovée, M. J. (2013). Body size over-estimation in women with anorexia nervosa is not qualitatively different from female controls. Body image, 10(1), 103-111.

de Vignemont, F. (2010). Body schema and body image-Pros and cons. Neuropsychologia, $48(3), 669-680$.

De Vignemont, F. (2018). Mind the body: An exploration of bodily self-awareness. Oxford University Press.

Engel, M., Gadsby, S., Corcoran, A. W., Keizer, A., Dijkerman, H. C., \& Hohwy, J. (2021). Waiting longer, feeling fatter: Effects of response delay on tactile distance estimation and confidence in females with anorexia nervosa.

Engel, M. M., \& Keizer, A. (2017). Body representation disturbances in visual perception and affordance perception persist in eating disorder patients after completing treatment. Scientific reports, 7(1), 1-9.

Espeset, E. M., Gulliksen, K. S., Nordbø, R. H., Skårderud, F., \& Holte, A. (2012). Fluctuations of body images in anorexia nervosa: patients' perception of contextual triggers. Clinical psychology \& psychotherapy, 19(6), 518-530.

Espeset, E. M., Nordb $\varnothing$, R. H., Gulliksen, K. S., Skårderud, F., Geller, J., \& Holte, A. (2011). The concept of body image disturbance in anorexia nervosa: an empirical inquiry utilizing patients' subjective experiences. Eating disorders, 19(2), 175-193.

Farrell, C., Lee, M., \& Shafran, R. (2005). Assessment of body size estimation: A review. European Eating Disorders Review: The Professional Journal of the Eating Disorders Association, 13(2), 75-88. 
Firestone, C. (2013). On the origin and status of the "El Greco fallacy". Perception, 42(6), 672-674.

Firestone, C., \& Scholl, B. J. (2014). "Top-down" effects where none should be found: The El Greco fallacy in perception research. Psychological science, 25(1), 38-46.

Firestone, C., \& Scholl, B. J. (2016). Cognition does not affect perception: Evaluating the evidence for "top-down" effects. Behavioral and Brain Sciences, 39, 1-72.

Gadsby, S. (2017a). Distorted body representations in anorexia nervosa. Consciousness and cognition, 51, 17-33.

Gadsby, S. (2017b). Explaining body size beliefs in anorexia. Cognitive neuropsychiatry, 22(6), 495-507.

Gadsby, S. (2019). Body Representations and Cognitive Ontology: Drawing the boundaries of the body image. Consciousness and cognition, 74, 102772.

Gadsby, S., \& Hohwy, J. (2020). Why Use Predictive Processing to Explain Psychopathology? The Case of. In D. Mendonça, M. Curado, \& S. S. Gouveia (Eds.), The Philosophy and Science of Predictive Processing (1 ed., pp. 209-226). Bloomsbury Academic.

Gadsby, S., \& Williams, D. (2018). Action, affordances, and anorexia: body representation and basic cognition. Synthese, 195(12), 5297-5317.

Gardner, R. (2012). Measurement of perceptual body image. Encyclopedia of body image and human appearance, 2, 526-532.

Gardner, R. M., \& Boice, R. (2004). A computer program for measuring body size distortion and body dissatisfaction. Behavior Research Methods, Instruments, \& Computers, 36(1), 89-95.

Gardner, R. M., \& Bokenkamp, E. D. (1996). The role of sensory and nonsensory factors in body size estimations of eating disorder subjects. Journal of Clinical Psychology, 52(1), 3-15.

Gardner, R. M., \& Brown, D. L. (2014). Body size estimation in anorexia nervosa: a brief review of findings from 2003 through 2013. Psychiatry Research, 219(3), 407-410.

Gardner, R. M., \& Moncrieff, C. (1988). Body image distortion in anorexics as a non-sensory phenomenon: A signal detection approach. Journal of Clinical Psychology, 44(2), 101107.

Garner, D. M., \& Garfinkel, P. E. (1982). Body image in anorexia nervosa: Measurement, theory and clinical implications. The International Journal of Psychiatry in Medicine, 11(3), 263-284.

Garner, D. M., Garfinkel, P. E., Stancer, H. C., \& Moldofsky, H. (1976). Body image disturbances in anorexia nervosa and obesity. Psychosomatic medicine.

Gross, S., Chaisilprungraung, T., Kaplan, E., Menendez, J. A., \& Flombaum, J. (2014). Problems for the purported cognitive penetration of perceptual color experience and Macpherson's proposed mechanism.

Jogan, M., \& Stocker, A. A. (2014). A new two-alternative forced choice method for the unbiased characterization of perceptual bias and discriminability. Journal of Vision, 14(3), 20-20.

Keizer, A., Smeets, M. A., Dijkerman, H. C., Uzunbajakau, S. A., van Elburg, A., \& Postma, A. (2013). Too fat to fit through the door: first evidence for disturbed body-scaled action in anorexia nervosa during locomotion. PLOS one, 8(5).

Keizer, A., Smeets, M. A. M., Dijkerman, H. C., Van den Hout, M., Klugkist, I., Van Elburg, A., \& Postma, A. (2011). Tactile body image disturbance in anorexia nervosa. Psychiatry Research, 190(1), 115-120. 
Keizer, A., Smeets, M. A. M., Dijkerman, H. C., van Elburg, A., \& Postma, A. (2012). Aberrant somatosensory perception in Anorexia Nervosa. Psychiatry Research, 200(2-3), 530537.

Longo, M. R., Azañón, E., \& Haggard, P. (2010). More than skin deep: body representation beyond primary somatosensory cortex. Neuropsychologia, 48(3), 655-668.

Longo, M. R., \& Haggard, P. (2012). Implicit body representations and the conscious body image. Acta Psychologica, 141(2), 164-168.

McCloskey, M., \& Chaisilprungraung, T. (2017). The value of cognitive neuropsychology: The case of vision research. Cognitive Neuropsychology, 34(7-8), 412-419.

Metral, M., Guardia, D., Bauwens, I., Guerraz, M., Lafargue, G., Cottencin, O., \& Luyat, M. (2014). Painfully thin but locked inside a fatter body: abnormalities in both anticipation and execution of action in anorexia nervosa. BMC research notes, 7(1), 707.

Mölbert, S. C., Klein, L., Thaler, A., Mohler, B. J., Brozzo, C., Martus, P., Karnath, H.-O., Zipfel, S., \& Giel, K. E. (2017). Depictive and metric body size estimation in anorexia nervosa and bulimia nervosa: A systematic review and meta-analysis. Clinical psychology review, 57, 21-31.

Mondraty, N., \& Sachdev, P. (2011). "My Body Is My Template": Why Do People Suffering from Anorexia Nervosa See Their Bodies Differently? In V. R. Preedy, R. R. Watson, \& C. R. Martin (Eds.), Handbook of Behavior, Food and Nutrition (pp. 3257-3270). Springer New York. https://doi.org/10.1007/978-0-387-92271-3_203

Morgan, M. J. (2014). A bias-free measure of retinotopic tilt adaptation. Journal of Vision, 14(1), 7-7.

Morgan, M. J., Melmoth, D., \& Solomon, J. A. (2013). Linking hypotheses underlying Class A and Class B methods. Visual neuroscience, 30(5-6), 197-206.

Niedenthal, P. M., \& Wood, A. (2019). Does emotion influence visual perception? Depends on how you look at it. Cognition and Emotion, 33(1), 77-84.

Peelen, M. V., \& Downing, P. E. (2007). The neural basis of visual body perception. Nature reviews neuroscience, 8(8), 636-648.

Risso, G., Martoni, R., Erzegovesi, S., Bellodi, L., \& Baud-Bovy, G. (2020). Visuo-tactile shape perception in women with Anorexia Nervosa and healthy women with and without body concerns. Neuropsychologia, 149, 107635.

Schilder, P. (1935). The image and appearance of the human body (Vol. 163). Routledge.

Shafran, R., \& Fairburn, C. G. (2002). A new ecologically valid method to assess body size estimation and body size dissatisfaction. International Journal of Eating Disorders, 32(4), 458-465.

Slade, P. D. (1985). A review of body-image studies in anorexia nervosa and bulimia nervosa. Journal of Psychiatric Research, 19, 255-265.

Slade, P. D., \& Russell, G. (1973). Awareness of body dimensions in anorexia nervosa: Crosssectional and longitudinal studies. Psychological medicine, 3(2), 188-199.

Smeets, M. A. (1997). The rise and fall of body size estimation research in anorexia nervosa: A review and reconceptualization. European Eating Disorders Review: The Professional Journal of the Eating Disorders Association, 5(2), 75-95.

Smeets, M. A., Klugkist, I. G., van Rooden, S., Anema, H. A., \& Postma, A. (2009). Mental body distance comparison: a tool for assessing clinical disturbances in visual body image. Acta Psychologica, 132(2), 157-165. 
Smeets, M. A., \& Panhuysen, G. E. (1995). What can be learned from body size estimation? It all depends on your theory. Eating disorders, 3(2), 101-114.

Smeets, M. A., Smit, F., Panhuysen, G. E., \& Ingleby, J. D. (1997). The influence of methodological differences on the outcome of body size estimation studies in anorexia nervosa. British Journal of Clinical Psychology, 36(2), 263-277.

Spitoni, G. F., Serino, A., Cotugno, A., Mancini, F., Antonucci, G., \& Pizzamiglio, L. (2015). The two dimensions of the body representation in women suffering from Anorexia Nervosa. Psychiatry Research, 230(2), 181-188.

Stokes, D. (2013). Cognitive penetrability of perception. Philosophy Compass, 8(7), 646-663.

Stokes, D. (2014). Cognitive Penetration and the Perception of Art. dialectica, 68(1), 1-34.

Thompson, M. A., \& Gray, J. J. (1995). Development and validation of a new body-image assessment scale. Journal of personality assessment, 64(2), 258-269.

Tuschen-Caffier, B., Bender, C., Caffier, D., Klenner, K., Braks, K., \& Svaldi, J. (2015). Selective visual attention during mirror exposure in anorexia and bulimia nervosa. PLOS one, 10(12), e0145886.

Valenti, J., \& Firestone, C. (2019). Finding the "odd one out": Memory color effects and the logic of appearance. Cognition, 191, 103934. 